\title{
LA TEORIA DI SAATY E L' APPLICAZIONE SUL TIRRENO-BRENNERO
}

\author{
Valeria Sabia $^{1}$, Antonietta Ametrano ${ }^{1}$, and IRMA FACCENDA ${ }^{1}$ \\ ${ }^{1}$ Affiliation not available
}

\begin{abstract}
L' ANALYTIC HIERARCHY PROCESS, nasce dal matematico Thomas Saaty, negli anni Settanta per organizzare ed analizzare decisioni complesse.

La metodologia consente di confrontare più alternative in relazione ad una pluralità di criteri, di tipo quantitativo o qualitativo, e ricavare una valutazione globale per ciascuna di esse. Ciò permette di:

- ordinare le alternative secondo un asse di preferenza;

- selezionare l'alternativa globalmente migliore;

- assegnare le alternative a sottoinsiemi predefiniti.

I punti di forza principali sono il confronto a coppie delle alternative decisionali e la separazione fra importanza del criterio e impatto sulla decisione.

Il metodo è basato su una scala di comparazione, suddivisa in nove livelli, la cui base teorica è data dal teorema di Perron-Frobenius.

Riportiamo a seguire la documentazione relativa alla realizzazione del corridoio Tirreno -Brennero sul quale è stato applicato il metodo AHP, relativamente al confronto dei diversi corridoi stradali e la successiva scelta di quello a minimo impatto ambientale.
\end{abstract}




\section{Analytic Hierarchy Process}

AHP è l' acronimo di:

(Analytic) Analitico: separazione di un qualunque problema, in elementi costituenti fondamentali.

(Hierarchy) Gerarchia: decomposizione dettagliata del problema: la decisione viene scomposta in tre livelli.

(Process) Processo: una serie di azioni che conducono ad un risultato finale.

L'analisi è composta da tre parti: il problema (goal) che si sta cercando di risolvere, tutte le possibili soluzioni (alternative) e i criteri su cui si giudicheranno quest' ultime.

Data la rilevanza della problematica di comparazione, il teorema dell' AHP è accompagnato da una scala ad-hoc, strutturata su nove livelli di giudizio, che permette di rendere le decisioni maggiormente consistenti e lineari.

\section{L'AHP trova una delle sue basi teoriche in uno specifico teorema Perron-Frobenius}

Se A, è una matrice non negativa primitiva e indecomponibile (cioè, con tutti gli elementi maggiori o uguali a zero) allora:

-L'autovalore di modulo massimo della matrice A, è reale e positivo;

-L' autovettore corrispondente ha tutte le componenti positive.

Nella teoria dell' AHP si usa su una matrice di confronti fra coppie di possibili decisioni: poichè A è a componenti strettamente positive, esiste un autovettore $\mathrm{w}$ che si usa per ordinare le alternative del decisore, ottenendone così una classifica.

Consideriamo le alternative A1, A2, A3 e supponendo di avere un criterio di merito che genera il 
vettore $\mathrm{w}=(8,4,1)$. Questo significa che secondo il nostro criterio gradiamo A1 con intensità 8 , A2 con intensità 4 e A3 con intensità 1 . Se si è coerenti nell'applicazione dei punteggi contenuti in w potete facilmente confrontare le alternative a due a due: ad esempio A1 è 2 volte preferibile ad A2 (8/4=2) e il decisore gradisce A3 un quarto di quello che gradisce A2, giungendo così alla matrice A:

Il legame tra la matrice e il vettore, è dato dal prodotto Aw.

Nel nostro caso è pari a 3 e rappresenta il numero di alternative presenti.

A seconda della metodologia utilizzata per il calcolo dell'autovettore, può essere necessario effettuare una normalizzazione del vettore al fine di ottenere una classifica definitiva, rappresentata dal vettore nw :

La valutazione della consistenza del decisore, per ognuna delle matrici elaborate, si può ottenere tramite uno specifico indice, il CONSISTENCY INDEX .

Si tratta di un valore che è nullo per decisori perfettamente consistenti e cresce all'aumentare delle contraddizioni nelle valutazioni: di conseguenza, più basso è e meglio è. Più precisamente, è un indice, che misura l'inconsistenza decisionale, a tal proposito, un decisore consistente ha un indice CI, che non supera il $10 \%$ del valore di inconsistenza casuale.

\section{IL CASO STUDIO: IL CORRIDOIO TIRRENO BRENNERO}

La progettazione delle opere di completamento del corridoio autostradale Tirreno - Brennero (Ti. Bre.) risulta di fondamentale importanza per lo sviluppo del sistema infrastrutturale italiano in quanto consente di realizzare il collegamento tra la A22 “del Brennero" e la A12 "Genova - Rosignano". Il progetto del Ti.Bre. si inserisce all'interno del progetto europeo di realizzazione del 
Corridoio 5, l'asse di collegamento Ovest-Est.

Sono state presentate diverse soluzioni progettuali:

-Tracciato alto: prevede il completamento dell'A15 della Cisa con una bretella autostradale di circa $80 \mathrm{~km}$ che collega Parma con Verona;

-Tracciato basso: prevede il collegamento dell'A15 con Mantova tramite una bretella autostradale. Tale soluzione è stata scartata a vantaggio del Tracciato alto;

-Autostrada Lucca - Modena: prevede il collegamento diretto tra l'Alto Tirreno e la A22 del Brennero con un raccordo autostradale di $80 \mathrm{~km}$, metà dei quali realizzati in galleria; per tale motivo questo progetto trova opposizioni ambientali.

Allo scopo di costruire una mappa di sensibilità territoriale atta ad individuare le porzioni di territorio che, sulla base dei criteri e degli attributi considerati, meglio si prestano ad accogliere la nuova infrastruttura viene applicato l'AHP.

Sono stati individuati tre criteri principali:"acqua", "suolo e sottosuolo", "paesaggio".

Vengono considerati anche alcuni vincoli escludenti che individuano le porzioni di territorio ad altissima sensibilità ambientale e quindi meno idonee al passaggio della infrastruttura.

Una volta definiti i grid relativi agli attributi della struttura gerarchica è necessario procedere alla ricomposizione gerarchica per ottenere le mappe di sensibilità territoriale rispetto ai criteri: esse sono ottenute come combinazione lineare di quelle degli attributi dove i coefficienti sono rappresentativi del peso.

L'assegnazione dei pesi dei quattro attributi rispetto al criterio "Acqua" viene effettuata attraverso il metodo dei confronti a coppie costruendo una matrice di rango $4 \mathrm{i}$ cui elementi sono computati in base alla scala semantica di Saaty di cui deve essere verificata la consistenza imponendo che il rapporto di consistenza CR non superi una soglia convenzionale del 10\%. Nel caso in esame la 
matrice dei confronti a coppie, il calcolo dei pesi cui corrisponde un valore di consistenza CR pari a 0,009 .

Per ottenere la mappa di sensibilità finale è necessario assegnare ai tre criteri un peso rappresentativo della sua importanza rispetto all'obiettivo ed effettuare poi una combinazione lineare fra i corrispondenti strati informativi geografici, sovrapponendo poi i fattori escludenti che eliminano le aree dove il tracciato non può passare.

La parte finale dell'analisi consiste nel definire i diversi scenari di valutazione e ne sono stati identificati quattro:

-Equilibrato: viene attribuita la stessa importanza a tutti gli elementi della gerarchia;

-Protezione suolo: viene attribuita maggiore importanza a tutti gli attributi connessi al criterio suolo;

-Protezione risorse idriche: viene attribuita maggiore importanza al criterio acqua ed alla protezione della risorsa idrica superficiale e sotterranea;

-Protezione del paesaggio: viene attribuita maggiore importanza agli attributi connessi alla protezione della tipicità dei luoghi.

Dall'analisi dei diversi scenari di sensibilità ambientale è possibile confrontare l'andamento planimetrico dei quattro corridoi di minima sensibilità ognuno corrispondente ad uno specifico scenario.

\section{CONCLUSIONI}

Dal confronto, ben tre tracciati su quattro tendono a coincidere per buona parte del loro sviluppo, distanziandosi solo in alcune zone dell'area di studio in cui si dovranno affrontare specifiche problematiche ambientali. L'unico scenario che porta alla definizione di un corridoio completamente diverso dagli altri è quello dipendente dal criterio "acqua": ciò dipende dal fatto che l'area di studio si caratterizza dalla presenza di ben due zone naturali tutelate a livello ambientale. 


\section{References}

1.Mocenni, C. Il Metodo di analisi multicriterio. (UNIVERSITà DEGLI STUDI DI SIENA).

2.CAVALLO, P. R. O. F. S. S. A. B. I. C. E. ELEMENTI DELLA TEORIA DELLE DECISIONI. (UNIVERSITà DEGLI STUDI DI NAPOLI).

3.Alessandra LAPUCCI, A. S. A. N. T. U. C. C. I. e A. C. O. F. R. A. N. C. E. S. C. O. L'INDIVIDUAZIONE DI UN CORRIDOIO STRADALE DI MINIMO IMPATTO. (UNIVERSITà DEGLI STUDI DI PISA). 


\section{Figure Captions}

Figure 1. STRUTTURA GERARCHICA

Figure 2. SCALA DI COMPARAZIONE

Figure 3. ES. APPLICAZIONE DEL TEOREMA

Figure 4. VETTORE NORMALIZZATO

Figure 5. INDICE DI CONSISTENZA

Figure 6. CONFRONTO DEI QUATTRO TRACCIATI 


\section{Figures}

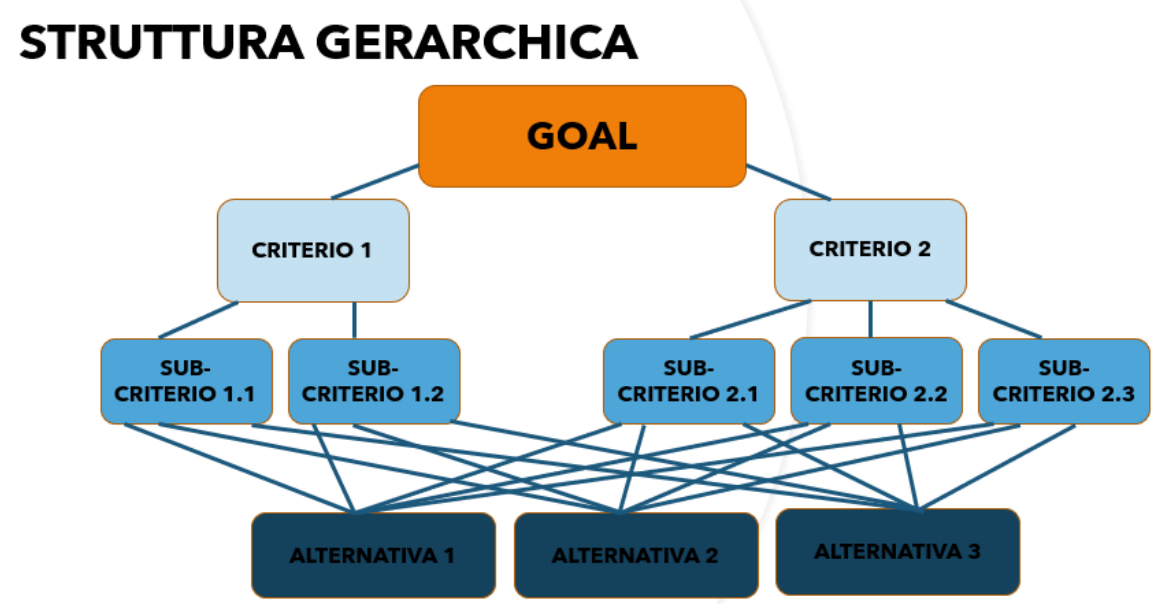

Figure 1: STRUTTURA GERARCHICA

\section{Scala di comparazione}

\begin{tabular}{|ll} 
INTENSITA' & GIUDIZIO \\
$\mid \begin{array}{l}1 \\
2\end{array}$ & Uguale importanza \\
\hline 3 & Debole importanza \\
4 & Moderata importanza \\
\hline 5 & Importanza più che moderata \\
6 & Importanza Forte \\
7 & Importanza più che forte \\
8 & Importanza notevolmente forte \\
9 & Estrema importanza
\end{tabular}

SPIEGAZIONE
Due attività contribuiscono egualmente
all'obiettivo
Situazione intermedia tra 1 e 3
L'esperienza e i giudizi favoriscono
leggermente un'attività rispetto all'altra
Situazione intermedia tra 3 e 5
Si favorisce fermamente un'attività rispetto
all'altra
Situazione intermedia tra 5 e 7
E' dimostrata nella pratica una dominanza
molto forte di un'attività sull'altra
Situazione intermedia tra 7 e 9
L'evidenza favorisce un'attività sull'altra al
massimo grado

Figure 2: SCALA DI COMPARAZIONE 


$$
A \mathbf{w}=\left(\begin{array}{ccc}
1 & 2 & 8 \\
\frac{1}{2} & 1 & 4 \\
\frac{1}{8} & \frac{1}{4} & 1
\end{array}\right)\left(\begin{array}{l}
8 \\
4 \\
1
\end{array}\right)=\left(\begin{array}{l}
8+8+8 \\
4+4+4 \\
1+1+1
\end{array}\right)=3\left(\begin{array}{l}
8 \\
4 \\
1
\end{array}\right)=3 \mathbf{w}
$$

Figure 3: ES. APPLICAZIONE DEL TEOREMA

$$
n \mathrm{w}=\frac{\mathrm{w}}{\sum \mathrm{wi}}
$$

Figure 4: VETTORE NORMALIZZATO

$$
C I=\frac{\lambda-n}{n-1}
$$

Figure 5: INDICE DI CONSISTENZA 


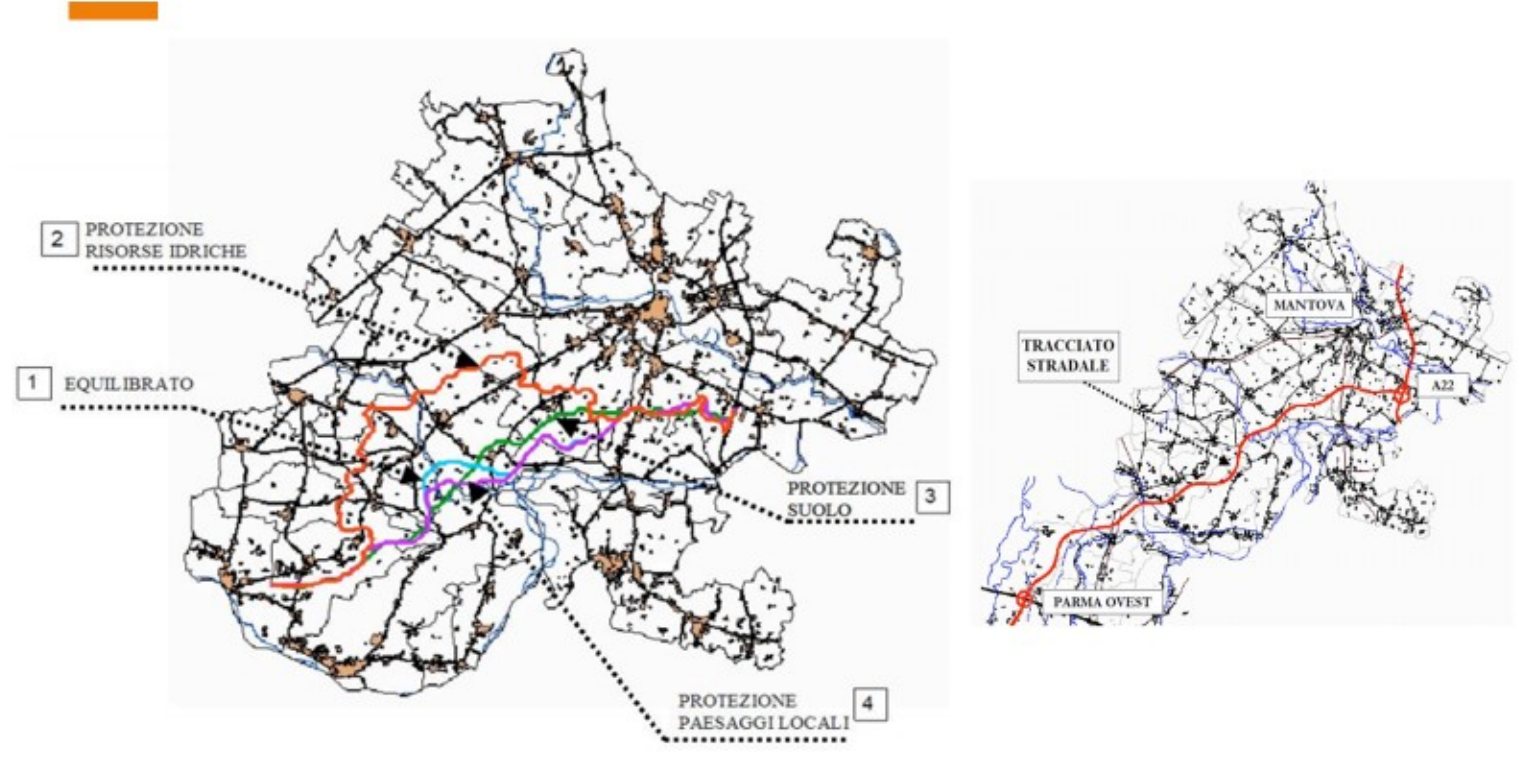

Figure 6: CONFRONTO DEI QUATTRO TRACCIATI 